\title{
Desenvolvimento in vitro do fungo simbionte de Atta sexdens rubropilosa Forel, 1908 (Hymenoptera: Formicidae) em meio de cultura com diferentes extratos vegetais
}

\author{
Development in vitro of the symbiotic fungus of Atta sexdens rubropilosa Forel, 1908 \\ (Hymenoptera: Formicidae) in culture medium with different plant extracts
}

\author{
Marcelo Dias de Souza' ${ }^{\mathrm{I}}$ Otávio Peres Filho ${ }^{\mathrm{II}}$ Sidney Fernando Caldeira ${ }^{\mathrm{II}}$ Alberto DorvalI \\ Nilton José Sousa
}

\section{RESUMO}

O objetivo deste estudo foi avaliar o desenvolvimento in vitro de Leucoagaricus gongylophorus, fungo simbionte de Atta sexdens rubropilosa, em meio de cultura acrescido com extratos de 24 espécies vegetais. O fungo foi inoculado no meio BDA, acrescido com os extratos vegetais, além de quatro controles, em placas de Petri. As placas foram mantidas em câmaras climatizadas à temperatura de $25 \pm 1^{\circ} \mathrm{C}$ no escuro, por um período de 42 dias. O crescimento do fungo foi avaliado semanalmente através do diâmetro da colônia. Os resultados mostraram que o fungo simbionte dessas formigas apresenta um crescimento diferenciado em relação aos extratos fornecidos nas placas, sendo que o meio acrescido com os extratos de arica (Physocalymma scaberrimum), cerejeira (Amburana acreana), cedro (Cedrela fissilis), timbó (Magonia pubescens), leucena (Leucaena leucocephala) $e$ mogno (Swietenia macrophylla) prejudicaram o desenvolvimento. No entanto, os extratos de genipapo (Genipa americana), seringueira (Hevea brasiliensis), ingá (Inga edulis) e cambará (Vochysia divergens) apresentaram melhores condições de desenvolvimento para o fungo simbionte.

Palavras-chave: crescimento, Leucoagaricus gongylophorus, formigas cortadeiras, simbiose.

\section{ABSTRACT}

The objective of this study was to evaluate the in vitro development of Leucoagaricus gongylophorus, the symbiotic fungus of Atta sexdens rubropilosa in culture medium added with extracts of 24 plant species. The fungus was inoculated in PDA added with vegetables extracts and four controls, in Petri dishes. The dishes were maintained in climate chambers at temperature of $25 \pm 1^{\circ} \mathrm{C}$ in the dark, to period of 42 days. The fungal growth was evaluated weekly by diameter of the colony. The results showed that the symbiotic fungus of these ants has differentiated growth when compared to the extracts provided in the dishes, and the medium supplemented with extracts of aricá (Physocalymma scaberrimum), cerejeira (Amburana acreana), cedro (Cedrela fissilis), timbó (Magonia pubescens), leucena (Leucaena leucocephala) e mogno (Swietenia macrophylla) hampered the development, however the extracts of genipapo (Genipa americana), seringueira (Hevea brasiliensis), ingá (Inga edulis) e cambará (Vochysia divergens) had better conditions for the development of the symbiotic fungus.

Key words: growth, Leucoagaricus gongylophorus, leaf-cutting ants, symbiosis.

\section{INTRODUÇÃo}

As formigas pertencentes à tribo Attini são conhecidas como formigas cortadeiras e têm recebido atenção especial dos pesquisadores pelos danos que causam às plantas silvestres e cultivadas (ZANETTI et al., 2002). As formigas cortadeiras dos gêneros Atta e Acromyrmex usam os vegetais frescos para o desenvolvimento de um fungo específico e para sua própria alimentação (DELLA LUCIA \& FOWLER, 1993; FERNANDES et al., 2002). Os fungos cultivados por essas formigas são basidiomicetos, pertencentes ao grupo G1, caracterizado pela presença de gongilídeos (CHAPELA et al., 1994), conhecido como

IPrograma de Pós-graduação em Engenharia Florestal, Universidade Federal do Paraná (UFPR), Rua Lothário Meissner 900, 80210-170, Jardim Botânico, Curitiba, PR, Brasil. E-mail: marcelo.dias@florestal.eng.br . Autor para correspondência. IIDepartamento de Engenharia Florestal, Universidade Federal do Mato Grosso (UFMT), Boa Esperança, Cuiabá, MT, Brasil. IIIDepartamento de Ciências Florestais, (UFPR), Curitiba, PR, Brasil. 
Leucoagaricus gongylophorus (Singer) Möller, identificado formalmente por Singer em 1986 (FISHER et al., 1994). O fungo cresce sobre o material vegetal, produzindo hifas e gongilídeos que constituem a única fonte de alimento para as larvas e fornecem cerca de 9\% da energia necessária para as operárias (QUINLAN \& CHERRETT, 1979).

Segundo BORBA et al. (2006), a preferência para o corte das espécies vegetais está relacionada com a exigência nutricional do fungo mutualista, já que, em um ecossistema natural, as formigas mostram preferência por algumas espécies vegetais, as quais são constantemente desfolhadas, enquanto outras não são atacadas, embora sejam abundantes e localizadas próximas ao ninho.

A capacidade dos diferentes substratos na promoção do desenvolvimento do fungo dessas formigas depende da sua composição química, que é diferente entre as espécies de plantas, sendo influenciada pelas condições culturais, fatores climáticos e estágio de desenvolvimento da planta (CASTELLANI et al., 2007). Essas diferenças afetam as preferências das formigas e o desenvolvimento do fungo, além de ser extremamente difíceis de serem medidas com precisão, mas são detectáveis pelo efeito na taxa de crescimento do fungo (MUDD \& BATEMAN, 1979).

Alguns autores realizaram trabalhos com extratos de plantas no desenvolvimento in vitro do fungo simbionte de várias espécies de formigas dos gêneros Atta eAcromyrmex. BORBA et al. (2006) concluíram que os meios de cultura acrescidos com extrato de tifa (Typha angustifólia L.) proporcionaram melhor desenvolvimento do fungo simbionte de Acromyrmex spp., em relação ao meio acrescido com extrato de azevém (Lolium multiflorum Lam.). CASTELLANI et al.(2009) constataram diferentes variações de crescimento do fungo simbionte de Atta capiguara Gonçalves, com extratos vegetais de Pennisetum purpureum Schum., Hyparrhenia rufa (Nees) Stapf., Brachiaria brizantha Stapf. cv., Saccharum officinarum L., e Paspalum sp., adicionados ao meio de cultura. Nesse contexto, o presente trabalho teve como objetivo verificar o efeito da adição de extratos de 24 espécies vegetais em meio de cultura BDA, no crescimento in vitro de $\mathbf{L}$. gongylophorus.

\section{MATERIAL E MÉTODOS}

O experimento foi realizado no Laboratório de Patologia Florestal da Faculdade de Engenharia Florestal (FENF) da Universidade Federal de Mato Grosso (UFMT), sendo que as formigas cortadeiras e o fungo simbionte usados no experimento foram coletados de formigueiros de Atta sexdens rubropilosa Forel (saúva-limão), presentes no Campus da UFMT em Cuiabá.
Fragmentos do fungo dos sauveiros, juntamente com algumas saúvas, foram retirados das panelas de fungo e armazenados em potes de plástico esterilizados e levados ao laboratório para o isolamento. Para obtenção dos extratos, foram coletadas folhas de 24 espécies florestais, que foram identificadas no laboratório de Dendrologia Florestal da UFMT (Tabela 1).

As folhas das espécies selecionadas foram secas em estufa a $40^{\circ} \mathrm{C}$ durante $48 \mathrm{~h}$, após esse processo foram trituradas em liquidificador. Os extratos foram obtidos através do processamento da massa seca das folhas com água destilada, na proporção de 1:6 (peso/ volume), sendo posteriormente filtrada através de tecido fino (voil) (SOUZA \& VENDRAMIM, 2001), onde $20 \mathrm{ml}$ de extrato foram transferidos imediatamente para $100 \mathrm{ml}$ de meio de cultura (BORBA et al., 2006; CASTELLANI et al., 2007).

Foi utilizado o meio de Cultura BDA, preparado com $200 \mathrm{~g}$ de batata, $20 \mathrm{~g}$ de dextrose e $15 \mathrm{~g}$ de ágar para $1000 \mathrm{ml}$ de água destilada. Os valores de pH dos meios foram ajustados para 5,5 com uma solução de $\mathrm{HCl} 1 \mathrm{~N}$ (diminuir) e $\mathrm{NaOH} 1 \mathrm{~N}$ (aumentar). Os frascos contendo o meio de cultura com adição dos extratos foram esterilizados em autoclave a $120^{\circ} \mathrm{C}$ e $1,1 \mathrm{~atm}$ de pressão, durante 15 minutos.

Em uma câmara asséptica de fluxo laminar, $15 \mathrm{ml}$ de meio foram transferidos para cada placa de Petri previamente esterilizadas $(90 \times 15 \mathrm{~mm})$, onde permaneceram até a completa solidificação. Para confirmar se o fungo isolado nas placas de Petri era o mesmo cultivado pelas saúvas, as placas contendo fungo foram fornecidas a sauveiros presentes no laboratório e observadas as reações das formigas, onde recolhiam o fungo e o incorporavam ao jardim da colônia (HOWARD et al., 1988; CAZIN JR. et al., 1989) e também pela observação de gongilídeos presentes no fungo isolado nas placas (Figura 1A)(CASTELLANI et al., 2007; MIYASHIRA et al., 2010), podendo assim iniciar o ensaio.

Preparados os tratamentos, discos de meio de cultura contendo micélio do fungo foram transferidos com um vazador de $5 \mathrm{~mm}$ de diâmetro, para as placas de Petri, que foram seladas com filme de polietileno e em seguida incubadas em câmaras climatizadas do tipo B.O.D à temperatura de $25 \pm 1^{\circ} \mathrm{C}$ em escotofase.

$\mathrm{O}$ crescimento do fungo foi avaliado macroscopicamente com base no diâmetro da colônia, em milímetros e em intervalos semanais, totalizando seis avaliações (aos 7, 14, 21, 28, 35 e 42 dias após inoculação). Para realização das mensurações, foram traçadas duas retas perpendiculares com ponto de cruzamento coincidindo com o centro do disco do inóculo (Figura 1B). As medidas foram realizadas a partir 
Tabela 1 - Espécies vegetais utilizadas para obtenção dos extratos incorporados no meio BDA destinados ao desenvolvimento in vitro do fungo Leucoagaricus gangylophorus.

\begin{tabular}{|c|c|c|}
\hline Nome vulgar & Espécie & Família \\
\hline Almecega & Protium heptaphyllum March & Burseraceae \\
\hline Angico branco & Albizia polycephala (Benth.) Killip & Fabaceae \\
\hline Aricá & Physocalymma scaberrimum Pohl. & Lythraceae \\
\hline Aroeira & Astronium urundeuva (Fr. All.) Engl.) & Anacardiaceae \\
\hline Caixeta & Simarouba versicolor St. Hil. & Simaroubaceae \\
\hline Cambará & Vochysia divergens Pohl. & Vochysiaceae \\
\hline Carvãozinho & Callisthene fasciculata Mart. & Vochysiaceae \\
\hline Cedro & Cedrela fissilis Vell. & Meliaceae \\
\hline Cerejeira & Amburana acreana (Ducke) A. C. Sm. & Leguminosae \\
\hline Copaíba & Copaifera langsdorffii Desf. & Caesalpinaceae \\
\hline Cumbarú & Dipteryx alata Vog. & Leguminosae \\
\hline Genipapo & Genipa americana L. & Rubiaceae \\
\hline Gonçaleiro & Astronium fraxinifolium Schott & Anacardiaceae \\
\hline Ingá & Inga edulis Mart. & Fabaceae \\
\hline Ipê-amarelo & Tabebuia áurea (Manso) Benth. \& Hook & Bignoniaceae \\
\hline Jatobá & Hymenaea courbaril L. & Leguminosae \\
\hline Jequitibá & Cariniana estrellensis (Raddi) Kuntze & Lecythidaceae \\
\hline Leucena & Leucaena leucocephala (Lam.) de Wit & Leguminosae \\
\hline Mogno & Swietenia macrophylla King & Meliaceae \\
\hline Mutamba & Guazuma tomentosa Kunth. & Sterculiaceae \\
\hline Pau-terra & Qualea grandiflora Mart. & Voquisiaceae \\
\hline Pequi & Caryocar brasiliensis Camb. & Caryocaraceae \\
\hline Seringueira & Hevea brasiliensis M. Arg. & Euphorbiaceae \\
\hline Timbó & Magonia pubescens St. Hil. & Sapindaceae \\
\hline
\end{tabular}

da borda do inóculo até a extremidade do crescimento do fungo no meio de cultura e, em seguida, calculado o valor médio dos diâmetros para cada placa.

O delineamento experimental foi inteiramente casualizado, sendo 28 tratamentos (quatro controles, BDA[Controle 1 e 3], BDA + Agua destilada sem Extrato [Controle 2 e 4] e 24 com BDA + extratos vegetais) e seis repetições. $\mathrm{O}$ experimento foi realizado em duas etapas, pois a BOD não comportava todos os tratamentos simultaneamente, todavia as condições de temperatura e fotoperíodo foram idênticas.

Os dados foram submetidos à análise de variância (ANOVA) e as médias comparadas pelo teste de Scott-Knott ao nível de 5\% de probabilidade de erro. As análises foram executadas através do software Assistat, versão 7.6 beta (SILVA \& AZEVEDO, 2002).

\section{RESULTADOS E DISCUSSÃO}

A variação do $\mathrm{pH}$ do meio de BDA após o preparo foi de 5,55 a 5,68 . Os extratos vegetais apresentaram uma faixa bem mais ampla, de 3,81 a 6,57, assim, após a mistura com o BDA, foram ajustados para 5,5 , onde houve redução no valor do $\mathrm{pH}$ entre 0,3 a 0,33 após a esterilização em autoclave (Tabela 2). Segundo CASTELLANI et al. (2007), com o valor de pH inicial de 4,5, há pouca variação após a autoclavagem, porém apresenta pouco crescimento micelial, quando comparado a pH de 6 e 7,5. Para LOECK et al. (2004), fungos cultivados pela tribo Attini apresentam um ótimo desenvolvimento em $\mathrm{pH}$ próximo de 5,0, já MUDD \& BATEMAN (1979) encontraram um valor de $\mathrm{pH}$ ideal a cerca de 5,5 a 6,0 para o fungo de Atta cephalotes Linnaeus.

Nos tratamentos BDA + extratos de aroeira, copaíba e mutanba não houve solidificação do meio e esses três tratamentos foram exluídos do ensaio, sendo possível observar que estes extratos apresentaram os menores valores de $\mathrm{pH}$, respectivamente, 3,81, 4,34 e 3,89.

Na primeira semana após o início do ensaio, já existiam diferenças significativas entre os crescimentos radiais do fungo nos diferentes tratamentos. Alguns extratos foram prejudiciais ao desenvolvimento e acabaram levando o fungo à morte em algumas placas ou apenas evitavam que o fungo se desenvolvesse por mais um período. Esse fato foi constatado nos tratamentos BDA + extratos de aricá, cerejeira, cedro, timbó, leucena e mogno, nos quais o desenvolvimento 


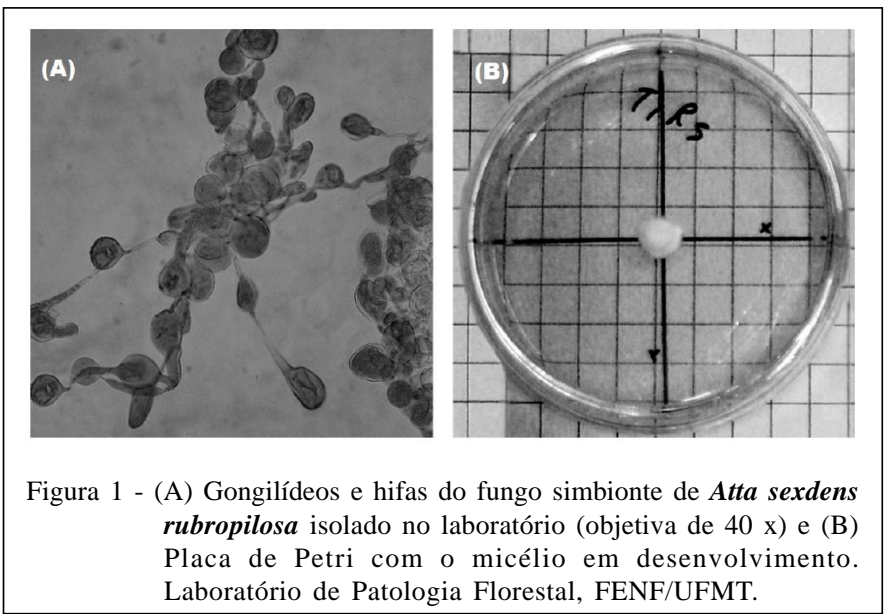

foi paralisado nos períodos 14, 14, 21, 21, 28 e 28, respectivamente (Tabela 3). Segundo CASTELLANI et al. (2009), ao incorporarem extratos de Cana de açúcar (Saccharum officinarum variedade SP80-1842) no meio de cultura BDA, houve prejuízo para o crescimento micelial do fungo simbionte de $A$. capiguara.
Os maiores diâmetros miceliais foram verificados nos tratamento BDA + extratos de cambará, seringueira, ingá e genipapo, sendo significativamente superiores aos demais tratamentos na maioria dos períodos avaliados (Tabela 3). CAMARGO et al. (2003) acrescentaram macerados de seis espécies de plantas

Tabela 2 - Valores de pH do meio BDA, do extrato vegetal, da mistura do BDA com o extrato após a correção, da mistura após a autoclavagem e da sua variação em relação ao BDA antes da autoclavagem.

\begin{tabular}{|c|c|c|c|c|c|c|}
\hline \multirow{2}{*}{ Meio } & \multicolumn{4}{|c|}{-----------------------------------------------pH } & \multicolumn{2}{|c|}{--------Variação-------- } \\
\hline & BDA & Extrato (E) & BDA + E Corrigido & BDA + E autoclavados & Valor & $(\%)$ \\
\hline BDA + Almécega & 5,68 & 4,93 & 5,41 & 5,08 & 0,33 & 6,10 \\
\hline BDA + Angico & 5,58 & 4,88 & 5,44 & 5,11 & 0,33 & 6,07 \\
\hline BDA + Aricá & 5,66 & 4,78 & 5,42 & 5,09 & 0,33 & 6,09 \\
\hline BDA + Aroeira & 5,58 & 3,81 & 5,47 & 5,26 & 0,21 & 3,84 \\
\hline BDA + Caixeta & 5,62 & 5,26 & 5,43 & 5,12 & 0,31 & 5,71 \\
\hline BDA + Cambará & 5,66 & 5,36 & 5,58 & 5,42 & 0,16 & 2,87 \\
\hline BDA + Carvãozinho & 5,67 & 5,17 & 5,46 & 5,28 & 0,18 & 3,30 \\
\hline BDA + Cedro & 5,63 & 6,16 & 5,62 & 5,54 & 0,08 & 1,42 \\
\hline BDA + Cerejeira & 5,66 & 6,12 & 5,61 & 5,58 & 0,03 & 0,53 \\
\hline BDA + Copíba & 5,63 & 4,34 & 5,43 & 5,13 & 0,30 & 5,52 \\
\hline BDA + Cumbarú & 5,68 & 5,18 & 5,38 & 5,27 & 0,11 & 2,04 \\
\hline BDA + Genipapo & 5,63 & 6,57 & 5,62 & 5,56 & 0,06 & 1,07 \\
\hline BDA + Gonçaleiro & 5,55 & 5,08 & 5,41 & 5,16 & 0,25 & 4,62 \\
\hline BDA + Ingá & 5,55 & 5,82 & 5,62 & 5,49 & 0,13 & 2,31 \\
\hline BDA + Ipê & 5,55 & 5,96 & 5,55 & 5,48 & 0,07 & 1,26 \\
\hline BDA + Jatobá & 5,55 & 5,89 & 5,61 & 5,43 & 0,18 & 3,21 \\
\hline BDA + Jequitibá & 5,62 & 4,89 & 5,39 & 5,10 & 0,29 & 5,38 \\
\hline BDA + Leucena & 5,58 & 5,12 & 5,43 & 5,26 & 0,17 & 3,13 \\
\hline BDA + Mogno & 5,68 & 4,52 & 5,32 & 5,06 & 0,26 & 4,89 \\
\hline BDA + Mutanba & 5.68 & 3.89 & 5,54 & 5,23 & 0,31 & 5,60 \\
\hline BDA + Pau-terra & 5,68 & 5,09 & 5,53 & 5,48 & 0,05 & 0,90 \\
\hline BDA + Pequi & 5,68 & 5,21 & 5,38 & 5,20 & 0,18 & 3,35 \\
\hline BDA + Seringueira & 5,62 & 5,85 & 5,66 & 5,59 & 0,07 & 1,24 \\
\hline BDA + Timbó & 5,62 & 4,58 & 5,45 & 5,15 & 0,30 & 5,50 \\
\hline
\end{tabular}

Ciência Rural, v.42, n.9, set, 2012. 
Tabela 3 - Diâmetro do crescimento micelial (mm) do fungo Leucoagaricus gongylophorus cultivado em meio de cultura BDA com diferentes extratos vegetais durante 42 dias.

\begin{tabular}{|c|c|c|c|c|c|c|}
\hline \multirow{2}{*}{ Tratamentos } & \multicolumn{6}{|c|}{-Período de incubação (dias)--- } \\
\hline & 7 & 14 & 21 & 28 & 35 & 42 \\
\hline BDA + Almécega & $5,3 \mathrm{~b}$ & $12,1 \mathrm{~b}$ & $18,3 \mathrm{~d}$ & $23,8 d$ & 30,3 e & $35,4 \mathrm{c}$ \\
\hline BDA + Angico & $1,7 \mathrm{~g}$ & $2,2 \mathrm{~g}$ & $2,5 \mathrm{~h}$ & $2,6 \mathrm{i}$ & $2,6 \mathrm{j}$ & $2,8 \mathrm{i}$ \\
\hline BDA + Aricá & $1,0 \mathrm{~h}$ & $1,2 \mathrm{~h}$ & - & - & - & - \\
\hline BDA + Caixeta & $1,4 \mathrm{~g}$ & $2,0 \mathrm{~g}$ & $2,5 \mathrm{~h}$ & $2,8 \mathrm{i}$ & $3,0 \mathrm{j}$ & $3,8 \mathrm{i}$ \\
\hline BDA + Cambará & 3,0 e & $14,1 \mathrm{a}$ & $24 \mathrm{a}$ & $30,6 \mathrm{~b}$ & $37,3 \mathrm{c}$ & $46,4 \mathrm{a}$ \\
\hline BDA + Carvãozinho & $2,4 \mathrm{f}$ & $7,8 \mathrm{~d}$ & $13 \mathrm{e}$ & $16,7 \mathrm{f}$ & $18,1 \mathrm{~h}$ & $21,4 \mathrm{~g}$ \\
\hline BDA + Cedro & $0,5 \mathrm{~h}$ & $0,6 \mathrm{~h}$ & $0,7 \mathrm{i}$ & - & - & - \\
\hline BDA + Cerejeira & $0,5 \mathrm{~h}$ & $0,5 \mathrm{~h}$ & - & - & - & - \\
\hline BDA + Cumbarú & 6,6 a & $14,2 \mathrm{a}$ & $21,5 \mathrm{~b}$ & $28,3 \mathrm{c}$ & $35,4 \mathrm{~d}$ & $40,7 \mathrm{~b}$ \\
\hline BDA + Genipapo & $3,3 \mathrm{~d}$ & 13,5 a & $23,2 \mathrm{a}$ & 32,6 a & $39,1 \mathrm{~b}$ & $44,8 \mathrm{a}$ \\
\hline Controle1 & $4,4 \mathrm{c}$ & 13,5 a & $19,7 \mathrm{c}$ & $23,4 d$ & 28,9 e & $34,1 \mathrm{c}$ \\
\hline Controle2 & $3,8 \mathrm{~d}$ & $11,5 \mathrm{~b}$ & $17,5 \mathrm{~d}$ & 21,9 e & $25,6 \mathrm{f}$ & $27,1 \mathrm{e}$ \\
\hline BDA + Gonçaleiro & $1,4 \mathrm{~g}$ & $10,1 \mathrm{c}$ & $18,5 \mathrm{~d}$ & 20,7 e & $21,9 \mathrm{~g}$ & $26,7 \mathrm{e}$ \\
\hline BDA + Ingá & $3,0 \mathrm{e}$ & 14,2 a & $23,0 \mathrm{a}$ & 32,4 a & $40,0 \mathrm{~b}$ & 45,4 a \\
\hline BDA + Ipê & $2,0 \mathrm{f}$ & $4,2 \mathrm{f}$ & $8,6 \mathrm{f}$ & $13,8 \mathrm{~g}$ & $17,3 \mathrm{~h}$ & $24,1 \mathrm{f}$ \\
\hline BDA + Jatobá & $1,9 \mathrm{f}$ & 9,6 c & $21,1 \mathrm{~b}$ & $29,5 \mathrm{~b}$ & $33,8 \mathrm{~d}$ & $38,8 \mathrm{~b}$ \\
\hline BDA + Jequitibá & $2,9 \mathrm{e}$ & $3,2 \mathrm{f}$ & $4,0 \mathrm{~g}$ & $4,4 \mathrm{~h}$ & $4,7 \mathrm{j}$ & $4,9 \mathrm{i}$ \\
\hline BDA + Leucena & $0,9 \mathrm{~h}$ & $1,1 \mathrm{~h}$ & $2,1 \mathrm{~h}$ & $2,4 \mathrm{i}$ & - & - \\
\hline BDA + Mogno & $0,8 \mathrm{~h}$ & $1,4 \mathrm{~h}$ & $1,5 \mathrm{i}$ & $1,7 \mathrm{i}$ & - & - \\
\hline BDA + Pau-terra & $1,6 \mathrm{~g}$ & $5,6 \mathrm{e}$ & 12,4 e & $14,1 \mathrm{~g}$ & $14,3 \mathrm{i}$ & $17,5 \mathrm{~h}$ \\
\hline BDA + Pequi & $2,2 \mathrm{f}$ & $3,9 \mathrm{f}$ & $4,4 \mathrm{~g}$ & $4,8 \mathrm{~h}$ & $5,0 \mathrm{j}$ & $5,1 \mathrm{i}$ \\
\hline BDA + Seringueira & $5,3 \mathrm{~b}$ & 13,9 a & $23,2 \mathrm{a}$ & $33,1 \mathrm{a}$ & 42,9 a & $45,7 \mathrm{a}$ \\
\hline BDA + Timbó & $2,3 \mathrm{f}$ & $2,5 \mathrm{~g}$ & $2,8 \mathrm{~h}$ & - & - & - \\
\hline Controle3 & $4,7 \mathrm{c}$ & $14,1 \mathrm{a}$ & $20,4 \mathrm{c}$ & $24,3 d$ & $26,6 \mathrm{f}$ & $30,4 d$ \\
\hline Controle4 & $4,1 \mathrm{~d}$ & $12,4 \mathrm{~b}$ & $18,2 \mathrm{~d}$ & $22,4 \mathrm{e}$ & $25,2 \mathrm{f}$ & $26,3 \mathrm{e}$ \\
\hline CV(\%) & 25,03 & 11,35 & 8,19 & 8,29 & 7,91 & 7,12 \\
\hline
\end{tabular}

Médias seguidas pela mesma letra nas colunas não diferem entre si pelo teste de Scott-Knott em nível de 5\% de probabilidade.

individualmente e uma mistura de todas no meio artificial (Ágar), na qual foram encontradas diferenças significativas entre os meios e o meio acrescido com Alchornea triplinervia (Spreng.), Müll. Arg. apresentou melhores condições de desenvolvimento, quando comparado com as outras espécies e até mesmo com o controle.

Diante dos dados obtidos, pode-se afirmar que a adição de alguns extratos vegetais incorporados ao meio BDA pode favorecer o desenvolvimento do fungo $\boldsymbol{L}$. gongylophorus, pois alguns dos meios acrescidos com os extratos apresentaram melhores condições de desenvolvimento que os controles. Segundo SIQUEIRA et al. (1998), o fungo simbionte de $\boldsymbol{A}$. sexdens cresce eficientemente em componentes encontrados nas folhas, como amido e xilana, sendo esses polissacarídeos, quando metabolizados pelo fungo, importantes fontes de carbono para a dieta das formigas.

BUENO et al. (1995) e HEBLING et al. (2000) observaram que componentes das folhas de algumas plantas exercem forte efeito sobre o desenvolvimento do fungo simbionte, beneficiando ou prejudicando o seu desenvolvimento, havendo evidências de que isso ocorra pela presença de metabólitos secundários presentes em algumas espécies. Sendo essa uma das possibilidades para explicar as alterações observadas em campo no comportamento de forrageio das formigas. 
Mesmo que a adição de alguns extratos possa beneficiar o desenvolvimento do fungo, ele ainda apresentou um crescimento lento em laboratório, o que corrobora muitos autores que analisaram o desenvolvimento in vitro do referido fungo (BORBA et al., 2006; CAMARGO et. al., 2003; CASTELLANI et al., 2007; CASTELLANI et al., 2009). Apesar das densidades de gongilídeos não terem sido quantificadas no presente trabalho, alguns meios apresentaram maiores quantidades de biomassa e quantidade reduzida de gongilídeos e outros apresentaram maiores quantidades de gongilídeos. Segundo CASTELLANI et al. (2007), certos meios de cultura podem resultar em maior produção de biomassa, especialmente hifas, no entanto com formação reduzida de gongilídeos.

\section{CONCLUSÃO}

De acordo com os resultados obtidos, podese concluir que os meios BDA contendo extratos de Physocalymma scaberrimum (aricá), Amburana acreana (cerejeira), Cedrela fissilis (cedro), Magonia pubescens (timbó), Leucaena leucocephala (leucena) e Swietenia macrophylla (leucena) prejudicaram o desenvolvimento do fungo em algum dos períodos, já os meios contendo os extratos de Genipa americana (genipapo), Hevea brasiliensis (seringueira), Inga edulis (ingá) e Vochysia divergens (cambará) proporcionaram melhor crescimento da colônia de fungo.

\section{AGRADECIMENTOS}

Ao Prof. Dr. Zenésio Finger do departamento de Engenharia Florestal, Universidade Federal do Mato Grosso (UFMT), pela identificação das espécies utilizadas na obtenção dos extratos. Aos Técnicos de Laboratório Manoel Lauro da Silva e Marcio Antônio Leão Pereira, pelo inestimável auxílio no desenvolvimento deste trabalho.

\section{REFERÊNCIAS}

BORBA, R.S. et al. Crescimento do fungo simbionte de formigas cortadeiras do gênero Acromyrmex em meios de cultura com diferentes extratos. Ciência Rural. v.36, n.3, p.725-730, 2006. Disponível em: <http://www.scielo.br/scielo.php?pid=S010384782006000300002\&script=sci_arttext $>$. Acesso em: 08 dez. 2010. doi: 10.1590/S0103-84782006000300002.

BUENO, O.C. et al. Effect of sesame (Sesamum indicum) on the nest development of Atta sexdens rubropilosa (Hymenoptera: Formicidae). Journal of Applied Entomology, v.119, p.341-343, 1995. Disponível em: <http:/ / onlinelibrary.wiley.com/doi/10.1111/j.14390418.1995.tb01297.x/abstract>. Acesso em: 20 mar. 2011. doi: 10.1111/j.1439-0418.1995.tb01297.x.

CAMARGO, R.S. et al. The effect of plant diversity on fungus garden development and foraging behavior of leaf-cutting ants
(Hymenoptera: Formicidae). Sociobiology, v.42, n2, p.110, 2003. Disponível em: <http://www.csuchico.edu/biol/ Sociobiology/volume/sociobiologyv42n22003.html\#11>. Acesso em: 10 mar. 2011.

CASTELLANI, M.A. et al. Isolation and growth of the symbiotic fungus of Atta capiguara (Hymenoptera: Formicidae). Sociobiology, v.50, n.3, p. 959-972, 2007. Disponível em: <http://www.csuchico.edu/biol/Sociobiology/volume/ sociobiologyv50n32007.html\#16>. Acesso em: $10 \mathrm{dez}$. 2010.

CASTELLANI, M.A. et al. Growth of the symbiotic fungus of the grass-cutting Ant Atta capiguara (Hymenoptera: Formicidae): effect of grass extracts. Sociobiology, v.54, n.1, p.283-298, 2009. Disponível em: <http://www.csuchico.edu/ biol/Sociobiology/volume/sociobiologyv54n12009.html\#24>. Acesso em: 20 dez. 2010.

CAZIN JR., J. et al. Isolation, growth characteristics, and long-term storage of fungi cultivated by Attini ants. Applied and Environmental Microbiology, v.55, n.6, p.1346-1350, 1989. Disponível em: <http://aem.asm.org/content/55/6/ 1346.full.pdf+html>. Acesso em: 25 abr. 2011.

CHAPELA, I.H. et al. Evolutionary history of the symbiosis between fungus-growing ants and their fungi. Science, v.266, n.5191 p.1691-1994, 1994. Disponível em: <http:// www.sciencemag.org/content/266/5191/1691>. Acesso em: 21 nov. 2010. doi: 10.1126/science.266.5191.1691.

DELLA LUCIA, T.M.C.; FOWLER, H.G. Formigas cortadeiras. In: DELLA LUCIA, T.M.C. (Ed.). As formigas cortadeiras. Viçosa: Folha de Viçosa, 1993. p.01-03, 262p.

FERNANDES, J.B. et al. Extrações de óleos de sementes de citros e suas atividades sobre a formiga cortadeira Atta sexdens e seu fungo simbionte. Química Nova, v.25, n.6, p.10911095, 2002. Disponível em: <http://dx.doi.org/10.1590/S010040422002000700009>. Acesso em: 05 abr. 2011. doi: 10.1590/ S0100-40422002000700009.

FISHER, P.J. et al. Leaf cutting ants, their fungus gardens and the formation of basidiomata of Leucoagaricus gongylophorus. Mycologist, v.8, n.3. p.541-546, 1994. Disponível em: <http:/ / w w w.sciencedirect.com/science/article/pii/ S0269915X09801596>. Acesso em: 18 ago. 2010. doi: 10.1016/S0269-915X(09)80159-6.

HEBLING, M.J.A. et al. Effects of leaves of Ipomoea batatas (Convolvulaceae) on nest development and on respiratory metabolism of leaf-cutting ants Atta sexdens L. (Hymenoptera, Formicidae). Journal of Applied Entomology, v.124, p.249252, 2000. Disponível em: <http://onlinelibrary.wiley.com/ doi/10.1046/j.1439-0418.2000.00471.x/full>. Acesso em: 03 out. 2010. doi: 10.1046/j.1439-0418.2000.00471.x.

HOWARD, J.J. et al. Toxicity of terpenoid deterrents to the leafcutting ant Atta cephalotes and its mutualistic fungus. Journal of Chemical Ecology, v.14, p.59-69, 1988. Disponível em: <http://www.springerlink.com/content/ u49w72h41338030l/>. Acesso em: 03 abr. 2011. doi: 10.1007/ BF01022531.

LOECK, A.E. et al. Growth of symbiont fungi of some higher Attini ants in mineral medium. Ciência Rural, v.34, n.1, p.79-82, 2004. Disponível em: <http://www.scielo.br/ 
scielo.php? script=sci_arttext\&pid=S $0103-$ 84782004000100012\&lng=en>. Acesso em: 16 dez. 2010. doi: 10.1590/S0103-84782004000100012.

MIYASHIRA, C.H. et al. Comparison of radial growth rate of the mutualistic fungus of Atta sexdens rubropilosa Forel in two culture media. Brazilian Journal of Microbiology, v.41, p.506-511, 2010. Disponível em: <http://www.scielo.br/scielo.php?pid=S1517$83822010000200035 \&$ script $=$ sci_arttext\&tlng=pt $>$. Acesso em: 14 mar. 2011. doi: 10.1590/S1517-83822010000200035.

MUDD, A.; BATEMAN, G.L. Rates of growth of the food fungus of the leaf-cutting ant Atta cephalotes (L.) (Hymenoptera: Formicidae) on different substrates gathered by the ants. Bulletin of Entomologic Research. v.69, p.141148, 1979. Disponível em: <http://journals.cambridge.org/ action/displayAbstract ? fromPage $=$ online $\&$ aid $=2425976>$. Acesso em: 15 fev. 2011. doi: 10.1017/S0007485300017971.

QUINLAN, R.J.; CHERRETT, J.M. The role of fungus in the diet of the leaf-cutting ant Atta cephalotes. Ecological Entomology, v.4, p.151-160, 1979. Disponível em: <http:// on linelibrary.wiley.com/doi/10.1111/j. 1365 2311.1979.tb00570.x/abstract>. Acesso em: 16 ago. 2010. doi: 10.1111/j.1365-2311.1979.tb00570.x.
SILVA, F.A.S.; AZEVEDO, C.A.V. Versão do programa computacional Assistat para o sistema operacional Windows. Revista Brasileira de Produtos Agroindustriais, v.4, n.1, p.71-78, 2002. Disponível em: <http://www.deag.ufcg.edu.br/ rbpa/rev41.html>. Acesso em: 08 abr. 2010.

SIQUEIRA, C.G. et al. Metabolism of plant polysaccharides by Leucoagaricus gongylophorus, the symbiotic fungus of the leafcutting ant Atta sexdens L. Applied and Environmental Microbiology, v.64, p.4820-4822, 1998. Disponível em: <http://aem.asm.org/content/64/12/4820.full>. Acesso em: 28 abr. 2011.

SOUZA, A.P.; VENDRAMIM, J.D. Atividade inseticida de extratos aquosos de Meliáceas sobre a Mosca-branca Bemisia tabaci (Genn.) biótipo B (Hemiptera: Aleyrodidae) Neotropical Entomology, v.30, n.1, p.133-137, 2001. Disponível em: <http://www.scielo.br/scielo.php?script=sci_arttext\&pid=S1519 566X2001000100019\&lng=en\&nrm=iso\&tlng=pt $>$. Acesso em: 20 mar. 2011. doi: 10.1590/S1519-566X2001000100019 .

ZANETTI, R. et al. Manejo integrado de formigas cortadeiras. Lavras: UFLA, 2002. 16p. 